Article

\title{
Integrated Phytochemical Analysis Based on UPLC-MS and Network Pharmacology Approaches to Explore the Quality Control Markers for the Quality Assessment of Trifolium pratense L.
}

\author{
Liyu Luo ${ }^{1}$, Wenya Gao ${ }^{2}$, Yan Zhang ${ }^{2}$, Chang Liu ${ }^{2}$, Guopeng Wang ${ }^{3}$, Hongwei Wu ${ }^{2, *}$ \\ and Wenyuan Gao ${ }^{1, *}$ \\ 1 School of Pharmaceutical Science and Technology, Tianjin University, Tianjin 300072, China; luoliyu@126.com \\ 2 Institute of Chinese Materia Medica, China Academy of Chinese Medical Sciences, Beijing 100700, China; \\ gaowenya0215@163.com (W.G.); 17862972336@163.com (Y.Z.); liuchangbj2009@126.com (C.L.) \\ 3 School of Chinese Materia Medica, Beijing University of Chinese Medicine, Beijing 100029, China; \\ binglelly@163.com \\ * Correspondence: whw9905012@163.com or hwwu@icmm.ac.cn (H.W.); pharmgao@tju.edu.cn (W.G.)
}

Received: 24 July 2020; Accepted: 14 August 2020; Published: 20 August 2020

\begin{abstract}
Red clover consists of the overground parts and inflorescence of Trifolium pratense L., a leguminous plant belonging to the genus Trifolium. It is widely distributed worldwide and has long been used in traditional medicine. In this study, a combination approach using UPLC-MS and network pharmacology was applied to explore the quality control markers for the quality assessments of red clover. Firstly, UPLC-MS was used to identify the compounds in different parts of red clover. Twenty-eight compounds were totally identified. According to the traditional clinical efficacy of red clover, a compound-target-function network was constructed by network pharmacology to discover the main active compounds based on the identified compounds. Nine compounds of chlorogenic acid, daidzin, calycosin-7-O- $\beta$-D-glucoside, genistin, ononin, daidzein, genistein, formononetin, and biochanin A were filtrated and further confirmed in rat plasma in view of the blood-absorbed components taking effects. Finally, a novel method for simultaneously detecting the nine quality control markers was developed by UPLC-QQQ-MS in an effort to assess the quality of red clover. For all samples, the average contents of the nine compounds measured from high to low consist of formononetin, ononin, biochanin A, genistin, daidzin, calycosin-7-O- $\beta$-D-glucoside, genistein, daidzein, and chlorogenic acid. The samples from Gansu province showed the best quality in the three producing areas This study provides new strategies to explore the quality control markers and develops a novel method for the quality assessment of red clover.
\end{abstract}

Keywords: red clover; network pharmacology; UPLC-MS/MS; quality control marker

\section{Introduction}

Red clover consists of the overground parts and inflorescence of Trifolium pratense L., a leguminous plant belonging to the genus Trifolium. It is widely distributed worldwide. In China, it can be found as a cultivated or wild plant, with its main distribution in the Gansu, Hubei and Shanxi provinces, and Northeast China [1]. Red clover has long been used in traditional medicine for the treatment of fever, diabetes, hepatitis, skin infections, snake bites, hypertension, and other diseases in several Asian countries, including China, India, and Thailand [2-5]. Modern pharmacological research has revealed that red clover has a variety of pharmacological activities including antiviral [6,7], anticancer [8-10], antibacterial [11,12], anti-inflammatory [13], antioxidant [14-17], and neuroprotective activities [18,19]. 
The major active components of red clover are considered to be flavones, isoflavones, coumarins, and volatile oil [20-22]. The isoflavones in red clover also have estrogen-like effects, so they are used to prevent osteoporosis [23,24], improve the symptoms of menopause [25,26], and heal wounds [27].

It is known that traditional herbal medicine is characterized by comprehensive medical effects with many components. The chemical markers of quality evaluation should reflect the overall efficacy. Therefore, how to select the quality control markers based on the clinical efficacy of herbal medicine is the key to evaluate the quality. As for red clover, although there are some reports about the quality evaluation of red clover using high-performance liquid chromatography (HPLC) [28,29], gas chromatography-mass spectrometry (GC-MS) [30,31], and liquid chromatography-mass spectrometry (LC-MS) [32,33], there are no reports using an integrated phytochemical analysis and network pharmacology approach to explore the quality control markers for the quality assessment of Trifolium pratense L.

In this study, UPLC-ESI-Orbitrap MS/MS was firstly used to identify the compounds in different parts of red clover. Through comparison with the reference substance and the fragmentation pattern analysis, a total of 28 main compounds were identified in the different parts, including stem, leaf and flower. Then, according to the traditional clinical efficacy of red clover and the identified compounds, a network pharmacology method was used to discovered the main active compounds and a "compound-target-function" network was constructed. Nine compounds of chlorogenic acid, daidzin, calycosin-7-O- $\beta$-D-glucoside, genistin, ononin, daidzein, genistein, formononetin, and biochanin A were selected as the potential quality control markers. Generally, traditional herbal medicine is administered orally, so the components those are absorbed into blood were believed to take effects. The nine potential quality control markers were further confirmed in the rat plasma by UPLC-MS. Finally, a novel method for simultaneously detecting the nine quality control markers was developed by UPLC-QQQ-MS and applied to assess the quality of 15 batches of the red clover samples with different origins.

\section{Results and Discussion}

\subsection{Metabolomics Profiling Analysis of Red Clover by UPLC-ESI-Orbitrap MS/MS}

A total of 28 components were identified from different parts of the red clover, including 24 isoflavones and 4 organic acids. Isoflavones include isoflavone aglycones (7 species), isoflavone glycosides (9 species), and isoflavone malonyl glucosides (8 species). Furthermore, the relative contents of the identified compounds in different parts of red clover were compared based on the response value of quasi-molecular ion peak. Their contents are generally low in stems, high in leaves and flowers, and the species are almost consistent. Detailed information including compound name, retention time, detected ion formula and high resolution mass spectrometry data are all listed in Table S1 of the Supplementary Materials.

As the identified components are mainly isoflavones, the characteristics of the fragmentation behaviors for isoflavones are summarized. Isoflavone aglycones usually undergo retro-Diels-Adel (RDA) reaction and loss of small molecule fragments through collision-induced dissociation. In isoflavone glycosides, glycosidic bonds are easily broken and they can lose glycosyl groups to generate aglycon ions. Malonyl glucosides usually lose malonyl groups to generate isoflavone glycosides. For example (as shown in Figure 1), the ion peak $[\mathrm{M}+\mathrm{H}]^{+}$of the biochanin A (isoflavone glycoside) in red clover has an $\mathrm{m} / \mathrm{z}$ of 285 . During collision-induced dissociation, the RDA reaction occurs and fragment ions of $m / z 153$ are generated. Small molecule fragments are also lost: A molecule of $\mathrm{CH}_{3}$ is lost to generate fragment ion $\mathrm{m} / \mathrm{z} 270$ due to methoxy bond breakage, and a carbonyl $\mathrm{CO}$ is lost to generate fragment ion $\mathrm{m} / \mathrm{z} 257$. Isoflavone glycosides often lose a glucose group during collision-induced dissociation. The formononetin excimer ion peak $[\mathrm{M}+\mathrm{H}]^{+}$has an $m / z$ of 269 . This ion loses one molecule of $\mathrm{CH}_{3}$ to generate the fragment ion with $\mathrm{m} / \mathrm{z} 254$, and loses one molecule of $\mathrm{CO}$ to generate fragment ion $m / z 241$, and is fragmented by RDA reaction to generate the fragment ion of $m / z 137$. The excimer ion peak $[\mathrm{M}+\mathrm{H}]^{+}$of the ononin (isoflavone glycoside) in red clover has a $\mathrm{m} / \mathrm{z}$ of 431 . 
Due to the glycosidic bond cleavage, a glucosyl group is lost, generating a fragment ion of $m / z 269$ and the other remaining fragment ions are consistent with formononetin. The calycosin-7-O- $\beta-\mathrm{D}-\mathrm{glucoside}$ $4^{\prime \prime}-O-$ malonate is found in red clover and shows a higher response signal in the positive ion mode than in negative ion mode, with an obvious $[\mathrm{M}+\mathrm{H}]^{+}$peak. An excimer ion peak with $m / z 533[\mathrm{M}+\mathrm{H}]^{+}$ appears in the first-order mass spectrometry in positive ion mode. The loss of the malonyl glucosyl group produces an ion with $m / z 285$, and $m / z 285$ loses one molecule of $\mathrm{CH}_{3}$ to generate the $\mathrm{m} / \mathrm{z} 270$ fragment ion. The fragment ion $m / z 137$ is generated by RDA cleavage.

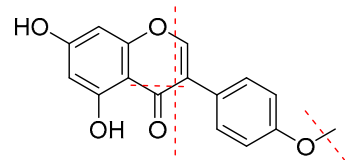

biochanin A

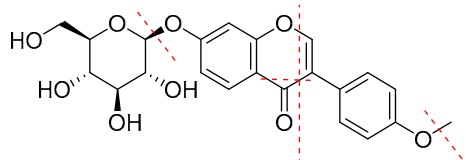

ononin

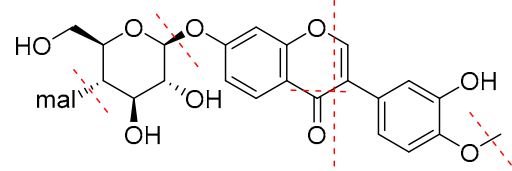

calycosin-7-O- $\beta$-D-glucoside 4 "-Omalonate

Figure 1. The fragmentation pathways of biochanin A, ononin and calycosin-7-O- $\beta$-D-glucoside $4 "$-O-malonate.

\subsection{Quality Control Markers Screening and Validation by Network Pharmacology and Blood-Absorbed Components Analysis}

A total of 93 high-confidence potential targets of the identified compounds from metabolomics profiling analysis were collected from BATMAN-TCM (http://bionet.ncpsb.org/batman-tcm/) databases (with the score cutoff $>20$ ). Using HPO databases, 294 proteins associated with the clinical effects of red clover were collected. Based on the above data, with the use of the STRING database for the retrieval of protein-protein interactions (PPI score $>0.8$ ), a total of 86 targets had high interaction associations and were finally reserved. These reserved proteins were further imported into the DAVID database (https://david.ncifcrf.gov/) for GO enrichment analysis. One of the enrichment analyses with a Benjamin $p$-value less than 0.05 was selected. Finally, the "component-target-function" network was visualized as shown in Figure 2 using Cytoscape 3.7.1 software. In Figure 2, there are a total of nine compounds (including chlorogenic acid, daidzin, calycosin-7-O- $\beta$-D-glucoside, genistin, ononin, daidzein, genistein, formononetin, and biochanin A), 86 proteins and 16 pathways.

Of the 16 key pathways, the three pathways of positive regulation of ERK1 and ERK2 cascade (GO: 0070374), inflammatory response (GO: 0006954), and negative regulation of inflammatory (GO: 0050728) response are related to anti-inflammatory effects, which was one of potential mechanisms for the preventing osteoporosis and healing wounds of red clover [34-36]. Positive regulation of cytosolic calcium ion concentration (GO: 0007204), calcium ion transmembrane transport (GO: 0070588), and calcium ion import (GO: 0070509) are all involved in the regulation of airway smooth muscle relaxation, which suggests the molecular mechanism of red clover in relieving cough and asthma [37]. In addition, positive regulation of cell growth (GO: 0030307) and negative regulation of apoptotic process (GO: 0043066) play a role in the treatment of burns and cardiovascular disease [38-40]. Oxidation-reduction process (GO: 0055114), one-carbon metabolic process (GO: 0006730), steroid biosynthetic process (GO: 0006694), and cyclooxygenase pathway (GO: 0019371) are related to the estrogen activity and anti-cancer activity which were reported for red clover [41-43]. The above results reveal the potential mechanisms of the filtered control markers for red clover. Furthermore, the nine potential quality control markers above were extracted by exact mass search in the rat plasma after oral administration of red clover, using the high accuracy of $m / z$ determined by UPLC-MS. All the nine compounds were successfully found and conformed by comparing their UPLC retention times and MS data with the authentic commercial standards. Detailed information can be seen in the Supplementary Materials. 


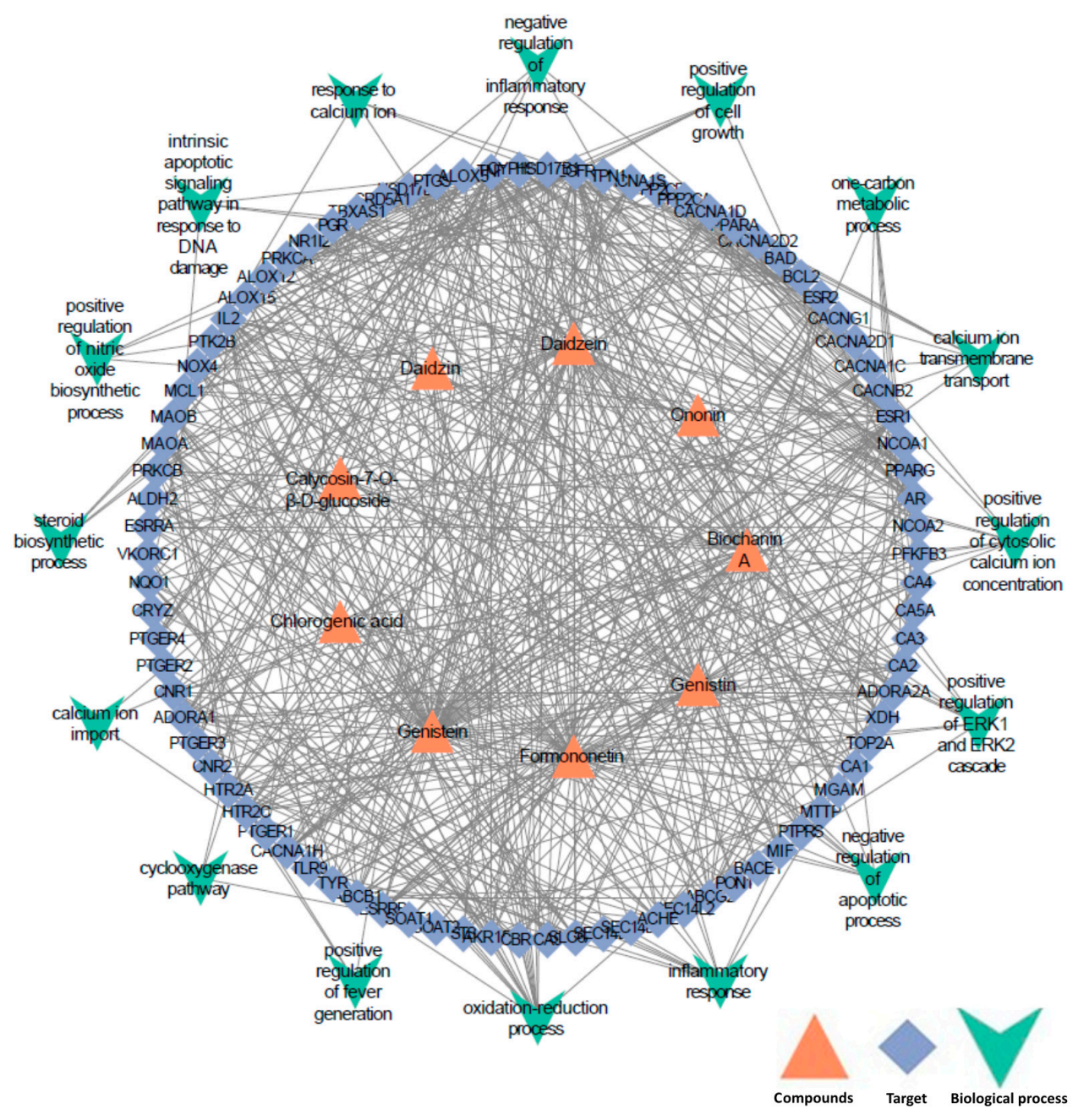

Figure 2. "Component-target-function" network for screening the quality control markers.

\subsection{UPLC-MS Method Validation}

\subsubsection{Specificity}

In this study, a UPLC-MS/MS method was established for simultaneous quantification of the nine compounds with a shortest possible runtime. Typical UPLC chromatograms of multiple reaction monitoring (MRM) for each detected compound are depicted in Figure 3. The specificity of this method was evaluated by comparing the chromatograms of the study samples, reagent blanks and the standards. No other components interfere with the detection of the target compounds. As shown in Figure 3, the nine compounds have ideal chromatographic separations and achieved good peak shape and symmetry factor under the chromatographic and mass spectrometric conditions as described in Section 3.6.2.

\subsubsection{Linearity and Limit of Quantitation}

Based on the content range of study samples from pre-assessment, a mixed standard stock solution $\left(\sim 15 \mu \mathrm{g} \cdot \mathrm{mL}^{-1}\right)$ was diluted to a series of suitable solution concentrations, which were then analyzed by UPLC-MS/MS. A calibration curve was generated using the peak area of each quantitative ion ( $Y$ value) and the corresponding mass concentration ( $X$ value, $n g / m L)$, then a linear equation and correlation coefficient were calculated. Good linear correlations for each compounds with $\mathrm{r}^{2}$ from 0.9991 to 1.0000 were obtained in the selected ranges. Characteristic parameters for the regression equations and correlation coefficients are given in Table 1. The limits of quantification (LOQ) were 
determined with the corresponding standard solution at a signal-to-noise $(\mathrm{S} / \mathrm{N})$ ratio of 10 for each component, respectively. The results show (Table 1) that LOQs of each compound meet the detection requirements and the method has suitable sensitivity.
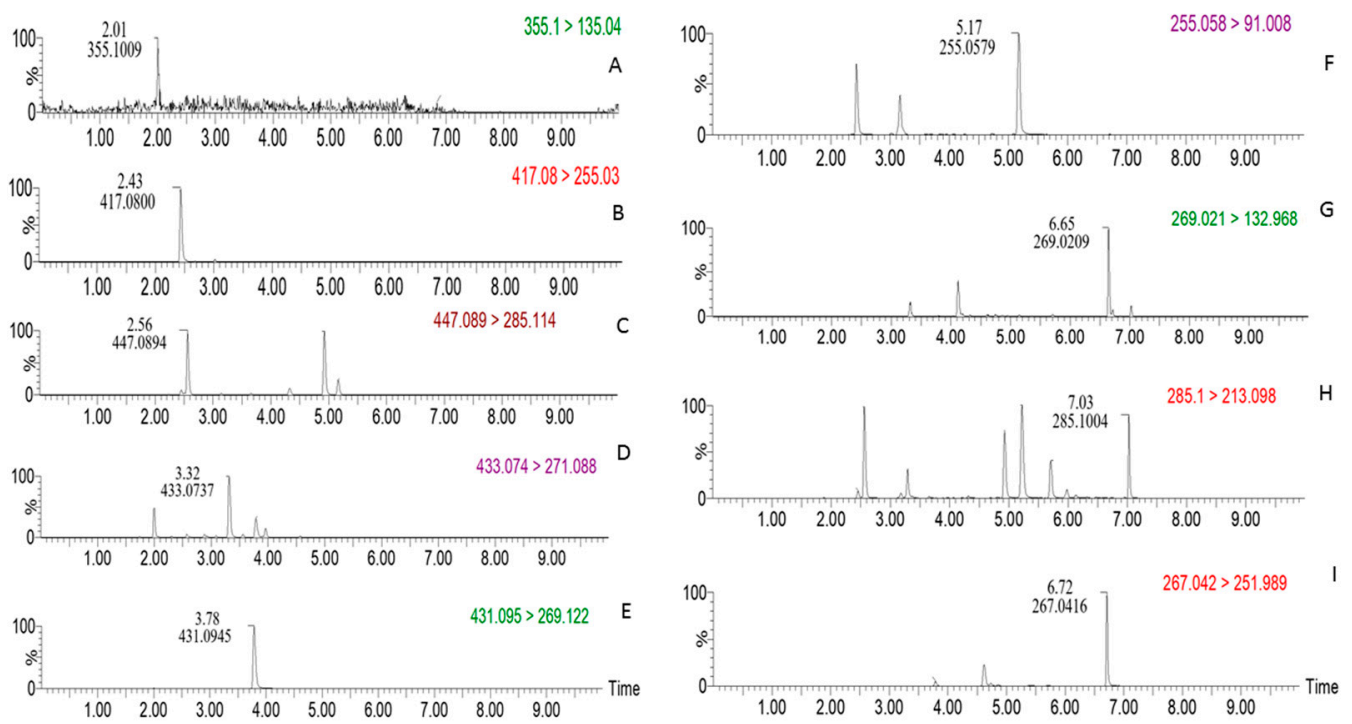

Figure 3. MRM (multiple reaction monitoring) chromatograms of the nine compounds detected in samples. (A): Chlorogenic acid; (B): daidzin; (C): calycosin-7-O- $\beta$-D-glucoside; (D): genistin; (E): ononin; (F): daidzein; (G): genistein; $(\mathbf{H})$ : biochanin $A$; $(\mathbf{I})$ : formononetin.

Table 1. Results of the method validation for the nine compounds detected in red clover.

\begin{tabular}{|c|c|c|c|c|c|c|c|}
\hline \multirow{2}{*}{ Compound Name } & \multirow{2}{*}{$\begin{array}{l}\text { Linear } \\
\text { Range } \\
(\mathrm{ng} / \mathrm{mL})\end{array}$} & \multirow[b]{2}{*}{$R^{2}$} & \multirow{2}{*}{$\begin{array}{l}\text { Limit of } \\
\text { Quantitation } \\
\text { (ng/mL) }\end{array}$} & \multirow{2}{*}{$\begin{array}{c}\text { Reproducibility } \\
\text { RSD } \\
(\%)(n=6)\end{array}$} & \multicolumn{3}{|c|}{ Average Recovery Rate (\%) } \\
\hline & & & & & $\begin{array}{l}\text { Low a } \\
(n=3)\end{array}$ & $\begin{array}{l}\text { Middle } \\
(n=3)\end{array}$ & $\begin{array}{c}\text { High } \\
(n=3)\end{array}$ \\
\hline Chlorogenic acid & $20.97 \sim 419.42$ & 0.9991 & 20.97 & 1.8 & 103.4 & 104.2 & 105.2 \\
\hline Daidzin & $1.45 \sim 289.14$ & 0.9995 & 0.014 & 2.5 & 96.5 & 105.2 & 97.8 \\
\hline $\begin{array}{l}\text { Calycosin-7-O- } \beta \text {-D- } \\
\text { glucoside }\end{array}$ & $1.28 \sim 255.43$ & 0.9999 & 0.013 & 2.8 & 96.6 & 103.5 & 103.5 \\
\hline Genistin & $1.20 \sim 239.08$ & 0.9997 & 0.24 & 3.8 & 97.8 & 101.4 & 97.3 \\
\hline Ononin & $1.66 \sim 165.58$ & 0.9998 & 0.017 & 1.5 & 100.4 & 104.1 & 97.7 \\
\hline Daidzein & $1.51 \sim 151.16$ & 0.9992 & 0.30 & 2.5 & 103.7 & 104.3 & 100.9 \\
\hline Genistein & $12.56 \sim 627.98$ & 0.9993 & 12.56 & 2.1 & 108.9 & 101.3 & 97.0 \\
\hline Formononetin & $1.17 \sim 233.16$ & 0.9997 & 0.50 & 1.2 & 102.5 & 95.4 & 97.5 \\
\hline Biochanin A & $1.77 \sim 884.35$ & 0.9997 & 0.71 & 2.2 & 104.7 & 103.4 & 97.5 \\
\hline
\end{tabular}

\subsubsection{Linearity and Limit of Quantitation Precision, Repeatability and Stability}

Six consecutive injections of the same sample solution were performed and the relative standard deviation (RSD) values of the peak areas for each compound were between $1.2 \%$ and $3.0 \%$, indicating good precision.

To confirm the repeatability, six parallel prepared samples from the same source of red clover were analyzed. The RSD of the contents for the nine compounds detected was $1.2-3.8 \%$, indicating that the method was repeatable.

In order to examine the stability of the sample, a single sample solution stored at room temperature $\left(25 \pm 3{ }^{\circ} \mathrm{C}\right)$ was analyzed respectively at $0,2,4,8,12$, and $24 \mathrm{~h}$ after preparation. The RSDs of the peak areas for each compound were between $0.8 \%$ and $3.9 \%$, indicating that the sample solution is stable within a 24-h period. 


\subsubsection{Accuracy}

The accuracy of the method was determined by spiking a known amount of mixed standards in known red clover samples in triplicate at levels 50\% (low), 100\% (middle) and 200\% (high) of the specified limit. Then the fortified samples were extracted, disposed as described above and analyzed with the procedure. The recoveries were estimated by the formula: Recovery $=$ (amount found original amount)/amount spiked. The recoveries of nine analytes were calculated and given in Table 1. The recovery of the investigated components ranged from $95.4 \%$ to $108.9 \%$ and their RSD values were all less than $5.0 \%$, characterizing good reliability and accuracy of the method.

\subsection{Determination of the Nine Quality Control Markers in 15 Batches of Red Clover}

The developed UPLC-MS/MS method was applied to the simultaneous determination of nine quality control markers in collected 15 red clover samples. Furthermore, the total contents of the nine compounds was also calculated. The results are presented in Table 2.

To investigate the global variations of the quality, we first used principal component analysis (PCA) to analyze all the samples based on the contents of nine components and their total content by SIMCA software (version 13.0). PCA, as an unsupervised pattern recognition method, can reflect the overall quality difference based on all the imported content indexes. As shown in the PCA score 3D plot (Figure 4A), an overview of all samples in the data can be observed and exhibits a clear grouping trend $\left(R^{2} X[1]=0.438 ; R^{2} X[2]=0.224, Q 2=0.291\right)$ between Gansu group (S1-S5), Hubei group (S6-S10) and Shanxi group (S11-S15). This observation indicates that there is an overall quality difference between the samples from different origins. In the loading scatter plot of PCA (Figure 4B), variables (compounds) situated far away from the origin (on the positive or negative side), especially such as genistin, ononin, total content, dominate the projection. In addition, variables near each other are positively correlated. As shown in Table 2, although the average content of formononetin was the highest in the detected compounds for all the samples, there were no significant differences between the samples from the three producing areas ( $p<0.05, t$-test). Except for formononetin, the average contents of all other components and the total content are the highest in the samples from Gansu province. The results indicated that the samples from Gansu province had the best quality in the three producing areas.

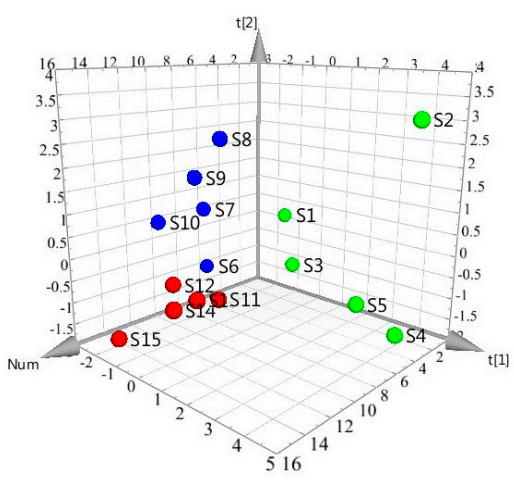

A

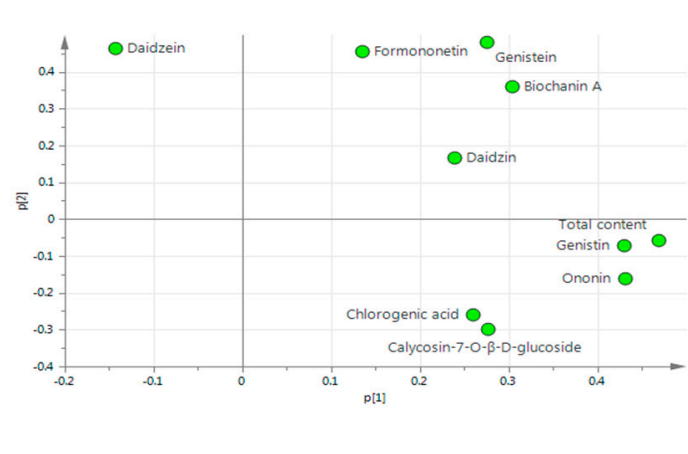

B

Figure 4. Principal component snalysis. (A): Three-dimensional (PCA 3D) score plots using the contents of the nine compounds and the total contents. S1-S5 (green), samples from Gansu; S6-S10 (blue), samples from Hubei; S11-S15 (red), samples from Shanxi. (B): Loadings scatter plot. 
Table 2. The contents of the 9 quality control markers in 15 batches of red clover.

\begin{tabular}{|c|c|c|c|c|c|c|c|c|c|c|c|}
\hline \multirow{2}{*}{ Sample Number } & \multicolumn{10}{|c|}{ Content of Compounds in Red Clover Extract (ng/mL) } & \multirow{2}{*}{ Location } \\
\hline & Chlorogenic Acid & Daidzin & Calycosin-7-O- $\beta$-D-glucoside & Genistin & Ononin & Daidzein & Genistein & Formononetin & Biochanin A & Total Content & \\
\hline S1 & 85 & 7361 & 3450 & 5175 & 33647 & 5662 & 3933 & 35193 & 11729 & 106235 & \multirow{6}{*}{ Gansu } \\
\hline S2 & 1717 & 18395 & 571 & 28649 & 72213 & 4678 & 9323 & 48149 & 40433 & 224128 & \\
\hline S3 & 5710 & 2880 & 2812 & 7970 & 35019 & 3146 & 5696 & 34597 & 19793 & 117623 & \\
\hline S4 & 7695 & 11353 & 29628 & 18027 & 72187 & 3562 & 4823 & 50069 & 28870 & 226214 & \\
\hline S5 & 1291 & 7952 & 8447 & 24379 & 80374 & 2342 & 4072 & 36096 & 26756 & 191709 & \\
\hline Average (S1-S5) & 3300 & 9588 & 8982 & 16840 & 58688 & 3878 & 5569 & 40821 & 25516 & 173182 & \\
\hline S6 & 397 & 5767 & 2202 & 3790 & 14945 & 4058 & 2233 & 22354 & 4359 & 60105 & \multirow{6}{*}{ Hubei } \\
\hline S7 & 739 & 12847 & 3094 & 3065 & 16570 & 6484 & 3280 & 44849 & 5792 & 96720 & \\
\hline S8 & 872 & 8382 & 2674 & 6294 & 18462 & 6727 & 7860 & 57704 & 35219 & 144194 & \\
\hline S9 & 217 & 2834 & 1430 & 2326 & 16204 & 5783 & 6203 & 75028 & 45784 & 155809 & \\
\hline S10 & 209 & 7003 & 1650 & 3152 & 11080 & 5944 & 4183 & 36855 & 8209 & 78285 & \\
\hline Average (S6-S10) & 487 & 7367 & 2210 & 3725 & 15452 & 5799 & 4752 & 47358 & 19873 & 107023 & \\
\hline S11 & 4023 & 3955 & 5301 & 9726 & 39039 & 2501 & 4873 & 46977 & 34518 & 150913 & \multirow{6}{*}{ Shanxi } \\
\hline S12 & - & 5457 & 3254 & 6584 & 33063 & 3033 & 3734 & 43176 & 23020 & 121321 & \\
\hline $\mathrm{S} 13$ & - & 4155 & 5628 & 8145 & 60609 & 3268 & 5434 & 74243 & 20612 & 182094 & \\
\hline S14 & - & 4701 & 5162 & 12056 & 47835 & 2475 & 4997 & 41490 & 19992 & 138708 & \\
\hline S15 & 1345 & 2142 & 2778 & 6023 & 24024 & 1854 & 3152 & 33998 & 15880 & 91196 & \\
\hline Average (S11-S15) & 2684 & 4082 & 4425 & 8507 & 40914 & 2626 & 4438 & 47977 & 22804 & 136846 & \\
\hline Average (S1-S15) & 2025 & 7012 & 5205 & 9691 & 38351 & 4101 & 4920 & 45385 & 22731 & 139017 & \\
\hline
\end{tabular}

Note: "-": indicates not detected. 
For all samples, the average contents of the nine compounds measured from high to low are of formononetin, ononin, biochanin A, genistin, daidzin, calycosin-7-O- $\beta$-D-glucoside, genistein, daidzein, and chlorogenic acid. In order to discover the potential relationship between the changes in the contents of these compounds, a Spearman correlation analysis was performed using the Software SPSS21.0 (SPSS Inc., Chicago, IL, USA). The Spearman correlation coefficient is the most commonly used measure of monotone association and it is usually suggested for non-normally distributed data. The closer the absolute value of the correlation coefficient ( $\mathrm{r}$ ) is to 1 , the more significant the correlation is. Generally, if the absolute value of the correlation coefficient was more than 0.5 , it indicated a reliable positive or negative correlation $(p<0.05)$.

The correlation coefficients between the nine compounds measured are summarized in Table S3 (in the Supplementary Materials). The correlations are depicted visually in Figure 5. Of the nine compounds' contents and the total contents, the significant correlations $(r>0.5$ and $p<0.05)$ are all positive. As shown in Figure 5, the total content with the most significant correlations are total content vs. calycosin-7-O- $\beta$-D-glucoside, total content vs. genistin, total content vs. ononin, total content vs. genistein, total content vs. formononetin, and total content vs. biochanin A. Secondly, except for chlorogenic acid, daidzin, and daidzein, the other compounds all have three significant correlations. These indicated that the results of the quality evaluation based on the compounds that have significant positive correlations are consistent. In addition, these correlations may be useful in providing evidence for or against specific biosynthetic pathways for red clover.

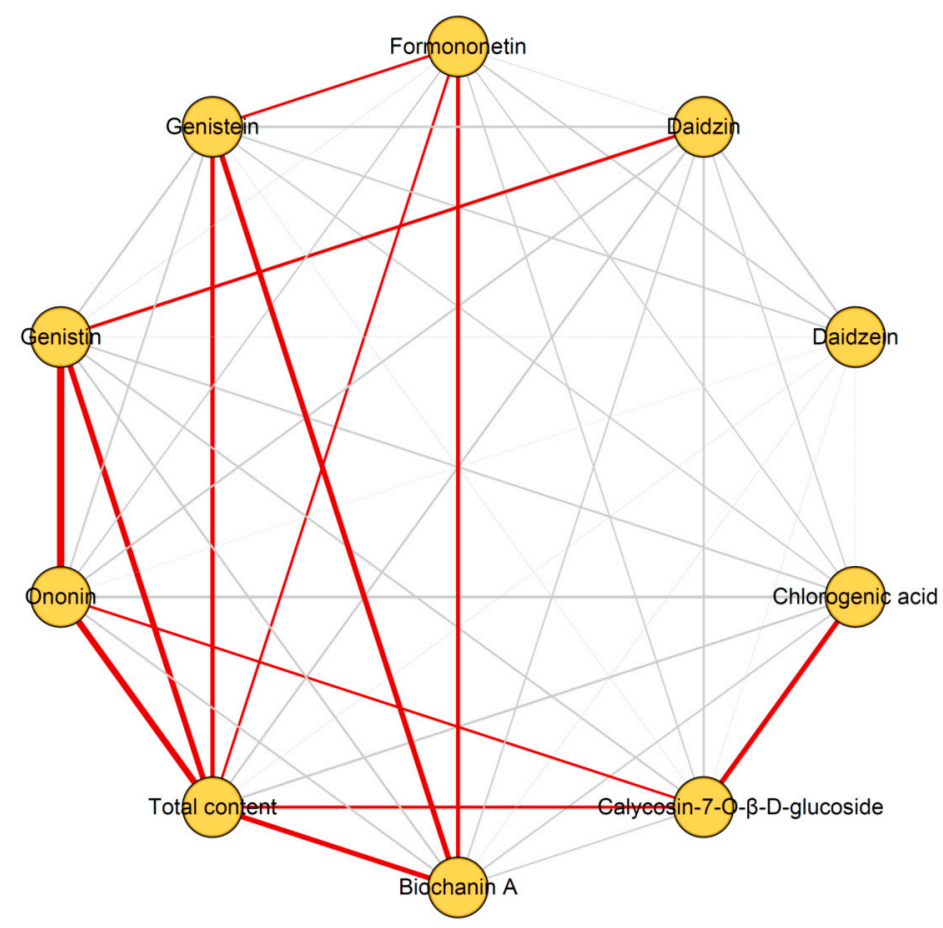

Figure 5. Correlation network for the contents of the nine compounds and the total contents detected in red clover. The significant correlations $(|r|>0.50$ and $p<0.05)$ are indicated with red lines. The positive correlations are indicated with solid line. The negative correlations are indicated with dotted lines. The weak correlations $(|r|<0.50)$ are indicated with grey lines. Thicker lines indicate a stronger correlation. The length of each line has no meaning. 


\section{Materials and Methods}

\subsection{Instruments and Chemicals}

An Ultimate 3000 Ultra High Performance Liquid Chromatography (UPLC) system coupled with LTQ-Orbitrap velos pro mass spectrometer was used (Thermo-Fisher, Waltham, MA, USA), as well as an Acquity UPLC I-Class UPLC system coupled with Xevo TQ-S micro triple quadrupole mass spectrometer (Waters, Milford, MA, USA).

The reference compounds of formononetin, ononin, daidzein, daidzin, genistin, genistein, calycosin-7-O- $\beta$-D-glucoside, biochanin A, and chlorogenic acid (purity $>98 \%$ for all) were all purchased from Shanghai Yuanye Biotechnology Co., Ltd. (Shanghai, China). Acetonitrile and formic acid were purchased from Fisher Scientific, San Jose, CA, USA Company (mass spec-grade). Deionized water was prepared by Milli-Q ultrapure water preparation system (Millipore Co., Ltd., Billerica, MA, USA). All other chemicals were purchased from Beijing Chemical Works (Beijing, China, analytical grade).

\subsection{Red Clover Sample Collection}

A total of 15 batches of red clover were collected from three main areas in the Gansu, Hubei, and Shanxi provinces in China. All the collected samples were denoted as follows: Gansu, S1 to S5; Hubei, S6 to S10; and Shanxi, S11 to S15. The samples were identified as the dry aboveground part (inflorescences, leaf and stems) of the legume Trifolium pratense L. by professor Wang Jingjuan of the Beijing University of Chinese Medicine. All samples were stored in a dry, constant environment to minimize any changes through degradation, and the voucher specimens were deposited in our laboratory.

\subsection{Preparation of Reference Solutions}

Accurately weigh an appropriate amount of each reference substance (5 10 mg) into a $50 \mathrm{~mL}$ volumetric flask, add methanol to dissolve and dilute to the mark, and obtain a single-component reference stock solution. Precisely measure an appropriate amount of each single-component reference stock solution into another $50 \mathrm{~mL}$ volumetric flask, dilute with methanol to the mark, and obtain a mixed reference stock solution with a concentration of $\sim 15 \mu \mathrm{g} \cdot \mathrm{mL}^{-1}$. Then, dilute the mixed reference stock solution to a series of mixed reference solutions at different concentrations.

\subsection{Metabolomics Profiling Analysis by UPLC-ESI-Orbitrap MS/MS}

\subsubsection{Sample Preparation for Compounds Identification}

The red clover was divided into flower, stem, and leaf portions. Then, $1.0 \mathrm{~g}$ powder of each part was accurately weighed and combined with $10 \mathrm{~mL}$ of $70 \%$ methanol, soaked for $1 \mathrm{~h}$ and ultrasonically extracted for $30 \mathrm{~min}$. The samples were finally filtrated through a $0.22 \mu \mathrm{m}$ membrane filter prior to the injection into UPLC-MS system.

\subsubsection{Chromatographic and Mass Spectrometric Conditions}

Instrument: Ultimate 3000 UPLC instrument, LTQ-Orbitrap velos pro mass spectrometer; column: ACQUITY UPLC BEH Shield RP18 $(2.1 \times 100 \mathrm{~mm}, 1.7 \mu \mathrm{m})$; flow rate: $0.3 \mathrm{~mL} / \mathrm{min}$; column temperature: $40{ }^{\circ} \mathrm{C}$; mobile phase: phase $\mathrm{A}-0.1 \%$ formic acid water, phase $\mathrm{B}$-acetonitrile; injection volume: $2 \mu \mathrm{L}$; gradient elution procedure: 0-1.0 $\mathrm{min}, 90-80 \% \mathrm{~A} ; 1-5.5 \mathrm{~min}, 80-60 \% \mathrm{~A} ; 5.5-6 \mathrm{~min}, 60-20 \% \mathrm{~A} ; 6-8 \mathrm{~min}$, 20-5\% A; 8-8.5 min, 5\% A; 8.5-9 $\min , 5-90 \%$ A; 9-10 $\min , 90-90 \%$ A.

The analysis was performed in positive and negative ion modes. Heater temperature: $350{ }^{\circ} \mathrm{C}$; capillary temperature: $350{ }^{\circ} \mathrm{C}$; capillary voltage: $35 \mathrm{~V}$; spray voltage: $3.4 \mathrm{kV}$; sheath gas $\left(\mathrm{N}_{2}\right)$ flow rate: 35 arb; auxiliary gas $\left(\mathrm{N}_{2}\right)$ flow rate: $10 \mathrm{arb}$; mass standard calibration using external standards (mass error is less than 5 ppm). The primary mass spectrum was scanned in FT mode (resolution R 
is 30,000, and the $\mathrm{m} / \mathrm{z}$ scan range is from 50 to 1500 ). The $\mathrm{MS}^{2}$ and $\mathrm{MS}^{3}$ spectra were obtained using a data-dependent scan. Dynamic ion exclusion mode was used to obtain additional compound information. Data acquisition and analysis were performed using Xcalibur, Metaworks, Mass Frontier 7.0 software (Thermo Fisher Scientific, San Jose, CA, USA).

\subsection{Quality Control Markers Screening and Validation by Network Pharmacology and Blood-Absorbed Components Analysis}

\subsubsection{Quality Control Markers Screening by Network Pharmacology}

The protocols included the following four main steps. (1) The potential targets of the all identified compounds in metabolomics profiling analysis were collected from BATMAN-TCM (http://bionet.ncpsb.org/batman-tcm/) databases [44]. In BATMAN-TCM, based on structural similarities between the drug molecules, the proteins with a score cutoff greater than 20 were selected as the potential targets. (2) According to clinical effects of red clover, such as preventing osteoporosis, improving the symptoms of menopause, relieving cough, and healing wounds, the potential targets related to diseases were collected by Human Phenotype Ontology (HPO, https://hpo.jax.org/app/). (3) The proteins of the drug molecular targets and the disease targets from the two databases were entered into the Search Tool (STRING database) for the retrieval of protein-protein interactions (PPI) [45]. Only when the PPI score was greater than 0.8 was the interaction confirmed, and the corresponding proteins and the related compounds were reserved. (4) The reserved proteins were imported into the DAVID database (https://david.ncifcrf.gov/) for GO enrichment analysis [46,47]. Then, one of the enrichment analyses with Benjamin p-value less than 0.1 was selected. Finally, the "component-target-function" network was visualized using Cytoscape 3.7.1 software (https://cytoscape.org/). In addition, in view of the blood-absorbed components taking effects, the potential quality control markers screened by network pharmacology were further analyzed and confirmed in rat plasma after oral administration of red clover by UPLC-MS.

\subsubsection{Blood-Absorbed Components Analysis for the Validation of Quality Control Markers}

$60 \mathrm{~g}$ of red clover powder was immersed in $600 \mathrm{~mL}$ of $70 \%$ Methanol for $1 \mathrm{~h}$, then heated and refluxed for $30 \mathrm{~min}$. After filtration, the extract was concentrated under reduced pressure and freeze-dried to obtain $7.8 \mathrm{~g}$ residue. Subsequently, the residue was dissolved in deionised water and the final concentration was $250 \mathrm{mg} \cdot \mathrm{mL}^{-1}$.

Male Sprague-Dawley rats (weighing $200 \pm 20 \mathrm{~g}$ ) were obtained from SPF (Beijing, China) Biotechnology Co., Ltd. The animals were acclimatized to laboratory conditions for 7 days and then fasted with free access to water for a $12 \mathrm{~h}$ period prior to the experiment. The rats were randomly divided into an experiment group $(n=6)$ and a blank group $(n=6)$. The extracted solution of red clover was administrated by oral administration at a single dose of $5 \mathrm{~g} \cdot \mathrm{kg}^{-1}$. The blood samples were collected from the orbital venous plexus in a heparinized tube at $0.5,1,2,3,4$, and $8 \mathrm{~h}$ after administration. The collected samples were then centrifuged at $4000 \mathrm{rpm}$ for $15 \mathrm{~min}$ at $4{ }^{\circ} \mathrm{C}$. The supernatant was removed and then mixed to obtain a pooled plasma. The blank plasma was collected in the same way. All experiments were performed in compliance with related laws and institutional guidelines, and the institutional committees approved the experiments.

A volume of $100 \mu \mathrm{L}$ of plasma sample was immediately treated with $400 \mu \mathrm{L}$ acetonitrile and then was vortexed for $3 \mathrm{~min}$ to precipitate plasma proteins. After centrifuging at $12,000 \mathrm{rpm}$ for $15 \mathrm{~min}$ at $4{ }^{\circ} \mathrm{C}$, the supernatants were evaporated to dryness under nitrogen gas at room temperature. The residue was reconstituted in $100 \mu \mathrm{L}$ methanol and centrifuged at $12,000 \mathrm{rpm}$ for $15 \mathrm{~min}$ at $4{ }^{\circ} \mathrm{C}$, followed by injection of $5 \mu \mathrm{L}$ into the analysis system. The instrument, chromatographic and mass spectrometric conditions were all the same with "3.4. Metabolomics Profiling Analysis by UPLC-ESI-Orbitrap MS/MS". 


\subsection{Development and Validation of UPLC-MS/MS Method for Quality Control of Red Clover}

\subsubsection{Sample Preparation for Quality Evaluation}

A total of $1.0 \mathrm{~g}$ powder of dried red clover was accurately weighed and placed into a $100 \mathrm{~mL}$ vial, with precisely $20 \mathrm{~mL}$ of $50 \%$ methanol added. The vial was weighed and recorded. Then, the sealed vial was extracted for $30 \mathrm{~min}$ by ultrasonication at room temperature. After cooling, 50\% methanol was added into the vial to make up to the initial weight. Then, $1 \mathrm{~mL}$ of supernatant fluid was diluted into a $25 \mathrm{~mL}$ volumetric flask and 50\% methanol was used to compensate the rest volume. Samples were finally filtrated through a $0.22 \mu \mathrm{m}$ membrane filter prior to injection into the UPLC-MS/MS system.

\subsubsection{Chromatographic and Mass Spectrometric Conditions}

The UPLC-MS/MS system consisted of an Acquity UPLC I-Class UPLC instrument (Waters, Milford, MA, USA) and Xevo TQ-S micro triple-quadrupole mass spectrometer. The separations were achieved on Agilent Extend C18 column $(3 \mathrm{~mm} \times 150 \mathrm{~mm}, 3.5 \mu \mathrm{m})$ and the column temperature was $25^{\circ} \mathrm{C}$. The mobile phase consisted of acetonitrile (A) and $0.1 \%$ formic acid solution (B), with the gradient elution of 0-23 $\mathrm{min}, 15-47 \%$ A; 23-25 $\mathrm{min}, 47-80 \%$ A; 25-26 min, 80-95\% A; 26-30min, 95-95\% A. The flow rate was $0.40 \mathrm{~mL} / \mathrm{min}$.

Quantification was performed using multiple reaction monitoring (MRM) based on positive and negative ion scanning. The precursor product ion transition and collision energies are listed in Table 3. The other parameters of the mass spectrometer were as follows: electrospray ionization source (ESI source); ion source temperature, $150{ }^{\circ} \mathrm{C}$; capillary voltage, $0.5 \mathrm{kV}$; desolvating gas, $\mathrm{N}_{2}$, flow rate, $1000 \mathrm{~L} / \mathrm{h}$; desolvating temperature, $500{ }^{\circ} \mathrm{C}$.

Table 3. Mass spectrometry parameters of the nine detected compounds.

\begin{tabular}{ccccccc}
\hline $\begin{array}{c}\text { Compound } \\
\text { Name }\end{array}$ & ESI & $\begin{array}{c}\text { Molecular } \\
\text { Formula }\end{array}$ & $\begin{array}{c}\text { Parent Ion } \\
(\mathbf{m} / \mathbf{z})\end{array}$ & $\begin{array}{c}\text { Daughter } \\
\text { Ion }(\mathbf{m} / \mathbf{z})\end{array}$ & $\begin{array}{c}\text { Cone } \\
\text { Voltage (V) }\end{array}$ & $\begin{array}{c}\text { Collision } \\
\text { Energy (eV) }\end{array}$ \\
\hline $\begin{array}{c}\text { chlorogenic acid } \\
\text { daidzin }\end{array}$ & + & $\mathrm{C}_{16} \mathrm{H}_{18} \mathrm{O}_{9}$ & 355.10 & 135.04 & 30 & 40 \\
$\begin{array}{c}\text { calycosin-7-O- } \beta \text {-D- } \\
\text { glucoside }\end{array}$ & + & $\mathrm{C}_{21} \mathrm{H}_{20} \mathrm{O}_{9}$ & 417.08 & 255.03 & 48 & 12 \\
$\begin{array}{l}\text { genistin } \\
\text { ononin }\end{array}$ & + & $\mathrm{C}_{22} \mathrm{H}_{22} \mathrm{O}_{10}$ & 447.09 & 285.11 & 52 & 16 \\
$\begin{array}{l}\text { daidzein } \\
\text { genistein }\end{array}$ & + & $\mathrm{C}_{21} \mathrm{H}_{20} \mathrm{O}_{10}$ & 433.07 & 271.09 & 24 & 16 \\
formononetin & - & $\mathrm{C}_{22} \mathrm{H}_{22} \mathrm{O}_{9}$ & 431.09 & 269.12 & 50 & 14 \\
biochanin $\mathrm{A}$ & - & $\mathrm{C}_{15} \mathrm{H}_{10} \mathrm{O}_{4}$ & 255.06 & 91.01 & 82 & 18 \\
\hline
\end{tabular}

\subsubsection{Methodology Validation}

The method was validated for specificity, precision, linearity, limit of quantification (LOQ), stability, repeatability and accuracy. The specificity of this method was evaluated by comparing the chromatograms of the study samples, reagent blanks and the standards. Linearity test solutions were prepared by diluting the mixed standards stock solution. The curve of regression between peak areas and concentration were calculated for the nine compounds. The LOQs for the nine compounds were estimated by injecting a series of dilute solutions at known concentration. Precision was determined by analyzing the same sample solution six times within one day. The sample stability was determined by analyzing a single sample solution that was stored at room temperature for $0,2,4,8,12$, and $24 \mathrm{~h}$. Repeatability was determined by analyzing six separate samples from the same source. The accuracy of the assay method was evaluated with the recovery of the standards from samples. Three different quantities (low, medium, high) of the authentic standards were added to the known real sample. The mixtures were extracted as described in Section 3.6.1 and were analyzed using the developed UPLC-MS/MS method. 


\section{Conclusions}

In this study, integrated phytochemical analysis and network pharmacology approaches were used to explore the quality control markers for the quality assessment of red clover. Firstly, UPLC-ESI-Orbitrap MS/MS was used to identify the compounds in different parts of red clover. A total of 28 compounds were indeed identified in stem, leaf and flower. The relative contents of the identified compounds are generally low in stems, high in leaves and flowers, and the species are almost consistent. Then, based on the traditional clinical efficacy of red clover and the identified compounds, a "compound-target-function" network was constructed using a network pharmacology method. Nine compounds of chlorogenic acid, daidzin, calycosin-7-O- $\beta$-D-glucoside, genistin, ononin, daidzein, genistein, formononetin, and biochanin A were selected as the potential quality control markers. Furthermore, the nine potential quality control markers were confirmed in the rat plasma by UPLC-MS. Finally, a novel method for simultaneously detecting the nine quality control markers was developed by UPLC-QQQ-MS and applied to assess the quality of 15 batches of the red clover samples with different origins. This study provides new strategies to explore the quality control markers and developed a novel method for the quality assessment of Trifolium pratense $\mathrm{L}$.

Supplementary Materials: The Supplementary Materials are available online.

Author Contributions: W.G. (Wenyuan Gao) conceived and designed the experiments; L.L. and W.G. (Wenya Gao) performed the experiments; Y.Z., G.W. and C.L. analyzed the data; L.L. and H.W. wrote the paper. All authors have read and agreed to the published version of the manuscript.

Funding: This study was financially supported by National Science and Technology Major Projects for "Major New Drugs Innovation and Development" (2019ZX09201005; 2019ZX09201-005-006-002).

Conflicts of Interest: The authors have no conflict of interest to declare.

\section{References}

1. Liu, Y.; Liu, S.H.; Wang, P. Research progress of Trifolium pratense. Chin. Herbal Med. 2007, 801-804.

2. Oza, M.J.; Kulkarni, Y.A. Trifolium pratense (Red Clover) Improve SIRT1 Expression and Glycogen Content in High Fat Diet-Streptozotocin Induced Type 2 Diabetes in Rats. Chem. Biodivers. 2020, 17, e2000019. [CrossRef] [PubMed]

3. Jiang, Y.B.; Yang, Y.R. Trifolium pratense isoflavones improve pulmonary vascular remodelling in broiler chickens. J. Anim. Physiol. Anim. Nutr. 2016, 100, 1159-1168. [CrossRef] [PubMed]

4. Circosta, C.; De Pasquale, R.; Palumbo, D.R.; Samperi, S.; Occhiuto, F. Effects of isoflavones from red clover (Trifolium pratense) on skin changes induced by ovariectomy in rats. Phytother. Res. 2006, 20, 1096-1099. [CrossRef]

5. Booth, N.L.; Piersen, C.E.; Banuvar, S.; Geller, S.E.; Shulman, L.P.; Farnsworth, N.R. Clinical studies of red clover (Trifolium pratense) dietary supplements in menopause: A literature review. Menopause 2006, 13, 251-264. [CrossRef] [PubMed]

6. Cecotti, R.; Carpana, E.; Bergomi, P.; Tava, A. Volatile constituents of Trifolium pratense spp. nivale quantified at different growth stages, and evaluation of their antimicrobial activity. Nat. Prod. Commun. 2013, 8, 1625-1628. [CrossRef]

7. Khazaei, M.; Pazhouhi, M. Protective effect of hydroalcoholic extracts of Trifolium pratense L. on pancreatic beta cell line (RIN-5F) against cytotoxicty of streptozotocin. Res. Pharm. Sci. 2018, 13, 324-331.

8. Jarred, R.A.; Keikha, M.; Dowling, C.; McPherson, S.J.; Clare, A.M.; Husband, A.J.; Pedersen, J.S.; Frydenberg, M.; Risbridger, G.P. Induction of apoptosis in low to moderate-grade human prostate carcinoma by red clover-derived dietary isoflavones. Cancer Epidemiol. Biomark. Prev. 2002, 11, 1689-1696.

9. Katz, A.E. Flavonoid and botanical approaches to prostate health. J. Altern. Complement Med. 2002, 8, 813-821. [CrossRef]

10. Atkinson, C.; Warren, R.M.; Sala, E.; Dowsett, M.; Dunning, A.M.; Healey, C.S.; Runswick, S.; Day, N.E.; Bingham, S.A. Red-clover-derived isoflavones and mammographic breast density: A double-blind, randomized, placebo-controlled trial [ISRCTN42940165]. Breast Cancer Res. BCR 2004, 6, R170-R179. [CrossRef] 
11. Flythe, M.; Kagan, I. Antimicrobial effect of red clover (Trifolium pratense) phenolic extract on the ruminal hyper ammonia-producing bacterium, Clostridium sticklandii. Curr. Microbiol. 2010, 61, 125-131. [CrossRef] [PubMed]

12. Dobrucka, R.; Dlugaszewska, J. Biosynthesis and antibacterial activity of $\mathrm{ZnO}$ nanoparticles using Trifolium pratense flower extract. Saudi J. Biol. Sci. 2016, 23, 517-523. [CrossRef] [PubMed]

13. Krenn, L.; Paper, D.H. Inhibition of angiogenesis and inflammation by an extract of red clover (Trifolium pratense L.). Phytomedicine 2009, 16, 1083-1088. [CrossRef] [PubMed]

14. Guo, Q.; Rimbach, G.; Moini, H.; Weber, S.; Packer, L. ESR and cell culture studies on free radical-scavenging and antioxidant activities of isoflavonoids. Toxicology 2002, 179, 171-180. [CrossRef]

15. Liu, B.J.; Guo, Y.S.; Qu, Y.P.; Diao, P.F. Study on the antioxidant effect of total flavonoids of Trifolium pratense in vitro. Chin. J. Pharm. 2009, 44, 1234-1237.

16. Kaurinovic, B.; Popovic, M.; Vlaisavljevic, S.; Schwartsova, H.; Vojinovic-Miloradov, M. Antioxidant profile of Trifolium pratense L. Molecules 2012, 17, 11156-11172. [CrossRef]

17. Arash, K.E.; Rosna, M.T.; Sadegh, M.; Behrooz, B. Antioxidant Activity and Total Phenolic and Flavonoid Content of Various Solvent Extracts from In Vivo and In Vitro Grown Trifolium pratense L. (Red Clover). Biomed Res. Int. 2015. [CrossRef]

18. Occhiuto, F.; Zangla, G.; Samperi, S.; Palumbo, D.R.; Pino, A.; De Pasquale, R.; Circosta, C. The phytoestrogenic isoflavones from Trifolium pratense L. (Red clover) protects human cortical neurons from glutamate toxicity. Phytomedicine 2008, 15, 676-682. [CrossRef]

19. Chen, H.Q.; Wang, X.J.; Jin, Z.Y.; Xu, X.M.; Zhao, J.W.; Xie, Z.J. Protective effect of isoflavones from Trifolium pratense on dopaminergic neurons. Neurosci. Res. 2008, 62, 123-130. [CrossRef]

20. Kami, T. Qualitative and quantitative analyses of the essential oils of red and Ladino white clovers. J. Agric. Food Chem. 1978, 26, 1194-1197. [CrossRef]

21. Vlaisavljevic, S.; Kaurinovic, B.; Popovic, M.; Djurendic-Brenesel, M.; Vasiljevic, B.; Cvetkovic, D.; Vasiljevic, S. Trifolium pratense L. as a potential natural antioxidant. Molecules 2014, 19, 713-725. [CrossRef] [PubMed]

22. Howes, J.B.; Howes, L.G. Content of isoflavone-containing preparations. Med. J. Aust. 2002, 176, $135-136$. [CrossRef] [PubMed]

23. Clifton-Bligh, P.B.; Baber, R.J.; Fulcher, G.R.; Nery, M.L.; Moreton, T. The effect of isoflavones extracted from red clover (Rimostil) on lipid and bone metabolism. Menopause 2001, 8, 259-265. [CrossRef] [PubMed]

24. Atkinson, C.; Compston, J.E.; Day, N.E.; Dowsett, M.; Bingham, S.A. The effects of phytoestrogen isoflavones on bone density in women: A double-blind, randomized, placebo-controlled trial. Am. J. Clin. Nutr. 2004, 79, 326-333. [CrossRef]

25. Coon, J.T.; Pittler, M.H.; Ernst, E. Trifolium pratense isoflavones in the treatment of menopausal hot flushes: A systematic review and meta-analysis. Phytomedicine 2007, 14, 153-159. [CrossRef]

26. Luís, Â.; Domingues, F.; Pereira, L.J.C. Effects of Red clover on perimenopausal and postmenopausal women's blood lipid profile: A meta-analysis. Climacteric 2018, 21, 446-453. [CrossRef]

27. Renda, G.; Yalçın, F.N.; Nemutlu, E.; Akkol, E.K.; Süntar, I.; Keleş, H.; Ina, H.; Çalış, I.; Ersöz, T. Comparative assessment of dermal wound healing potentials of various Trifolium L. extracts and determination of their isoflavone contents as potential active ingredients. J. Ethnopharmacol. 2013, 148, 423-432. [CrossRef]

28. Li, T.X.; Hu, Y.T.; Chu, C.S.; Gou, Q.X.; Chang, Y. Determination of six isoflavones in Trifolium pratense by one test and multiple evaluation method. Chin. Herbal Med. 2018, 41, 2619-2622.

29. Ma, Q.; Zhou, Y.X.; Lei, H.M.; Wang, C.H. Determination of daidzein, genistein, formononetin and biochanin A in Trifolium pratense L. by HPLC. Chin. Tradit. Pat. Med. 2005, 1193-1195.

30. He, L.; Zhou, P.; Wen, Z.H.; Zhang, G.P. Analysis of volatile components of Trifolium pratense from different habitats by HS-SPME-GC-MS. Chin. Herbal Med. 2018, 1122-1128.

31. He, C.L.; Zhang, G.P.; Wang, R.Y.; Zhou, P. HS-SPME-GC-MS and principal component analysis of volatile oil components in different parts of Trifolium pratense. Chin. J. Exp. Pharmacol. 2018, 24, 71-81.

32. Wu, Q.; Wang, M.; Simon, J.E. Determination of isoflavones in red clover and related species by high-performance liquid chromatography combined with ultraviolet and mass spectrometric detection. J. Chromatogr. A 2003, 1016, 195-209. [CrossRef] [PubMed]

33. Ma, Q.; Zhang, J.L.; Zhou, Y.X.; Lei, H.M.; Wang, C.H. Determination of isoflavones in Trifolium pratense by with high performance liquid chromatography electrospray ionization mass spectrometry. Anal. Chem. 2006, 34, 247-250. 
34. Occhiuto, F.; Pasquale, R.D.; Guglielmo, G.; Palumbo, D.R.; Zangla, G.; Samperi, S.; Renzo, A.; Circosta, C. Effects of phytoestrogenic isoflavones from red clover (Trifolium pratense L.) on experimental osteoporosis. Phytother. Res. 2007, 21, 130-134. [CrossRef] [PubMed]

35. Lee, S.A.; Moon, S.M.; Han, S.H.; Kim, J.S.; Kim, D.K.; Kim, C.S. The Effect of the Prethanol Extract of Trifolium pratense Leaves on Interleukin-1beta-Induced Cartilage Matrix Degradation in Primary Rat Chondrocytes. Cells Tissues Organs 2018, 206, 95-105. [CrossRef]

36. Ling, S.; Dai, A.; Williams, M.R.; Husband, A.J.; Nestel, P.J.; Komesaroff, P.A.; Sudhir, K. The isoflavone metabolite cis-tetrahydrodaidzein inhibits ERK-1 activation and proliferation in human vascular smooth muscle cells. J. Cardiovasc. Pharmacol. 2004, 43, 622-628. [CrossRef]

37. Sun, J.; Geng, T.; Pan, Q.; Xiao, F. Research progress of isoflavones in Trifolium pratense. Foreign Med. 2007, 22, 150-155.

38. Duan, H.; Chai, J.; Sheng, Z.; Yao, Y.; Yin, H.; Liang, L.; Shen, C.; Lin, J. Effect of burn injury on apoptosis and expression of apoptosis-related genes/proteins in skeletal muscles of rats. Apoptosis 2009, 14, 52-65. [CrossRef]

39. Teede, H.J.; McGrath, B.P.; DeSilva, L.; Cehun, M.; Fassoulakis, A.; Nestel, P.J. Isoflavones reduce arterial stiffness: A placebo-controlled study in men and postmenopausal women. Arterioscler. Thromb. Vasc. Biol. 2003, 23, 1066-1071. [CrossRef]

40. Reeve, J.L.; Duffy, A.M.; O'Brien, T.; Samali, A. Don't lose heart-therapeutic value of apoptosis prevention in the treatment of cardiovascular disease. J. Cell. Mol. Med. 2005, 9, 609-622. [CrossRef]

41. Locasale, J.W. Serine, glycine and one-carbon units: Cancer metabolism in full circle. Nat. Rev. Cancer 2013, 13, 572-583. [CrossRef] [PubMed]

42. Mueller, M.; Hobiger, S.; Jungbauer, A. Red clover extract: A source for substances that activate peroxisome proliferator-activated receptor alpha and ameliorate the cytokine secretion profile of lipopolysaccharide-stimulated macrophages. Menopause 2010, 17, 379-387. [CrossRef] [PubMed]

43. Lam, A.N.; Demasi, M.; James, M.J.; Husband, A.J.; Walker, C. Effect of red clover isoflavones on cox-2 activity in murine and human monocyte/macrophage cells. Nutr. Cancer 2004, 49, 89-93. [CrossRef]

44. Liu, Z.Y.; Guo, F.F.; Wang, Y.; Li, C.; Zhang, X.L.; Li, H.L.; Diao, L.H.; Gu, J.Y.; Wang, W.; Li, D.; et al. BATMAN-TCM: A Bioinformatics Analysis Tool for Molecular mechANism of Traditional Chinese Medicine. Sci. Rep. 2016, 6, 21146. [CrossRef] [PubMed]

45. Brohée, S.; Faust, K.; Lima-Mendez, G.; Vanderstocken, G.; van Helden, J. Network Analysis Tools: From biological networks to clusters and pathways. Nat. Protoc. 2008, 3, 1616-1629. [CrossRef] [PubMed]

46. Dennis, G., Jr.; Sherman, B.T.; Hosack, D.A.; Yang, J.; Gao, W.; Lane, H.C.; Lempicki, R.A. DAVID: Database for Annotation, Visualization, and Integrated Discovery. Genome Biol. 2003, 4, 1-11. [CrossRef]

47. da Huang, W.; Sherman, B.T.; Lempicki, R.A. Systematic and integrative analysis of large gene lists using DAVID bioinformatics resources. Nat. Protoc. 2009, 4, 44-57. [CrossRef]

Sample Availability: Samples of the compounds are available from the authors.

(C) 2020 by the authors. Licensee MDPI, Basel, Switzerland. This article is an open access article distributed under the terms and conditions of the Creative Commons Attribution (CC BY) license (http://creativecommons.org/licenses/by/4.0/). 